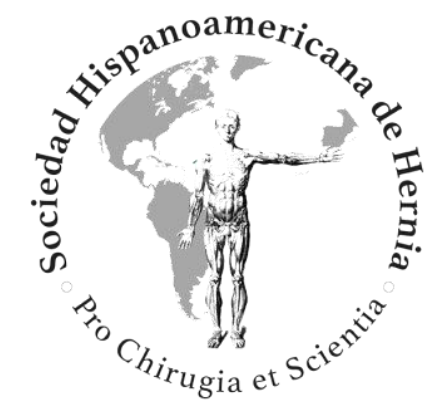

Neumoperitoneo preoperatorio en la preparación quirúrgica de las eventraciones complejas

\title{
Preoperative pneumoperitoneum in the surgical preparation of complex eventrations
}


Neumoperitoneo preoperatorio en la preparación quirúrgica de las eventraciones complejas

Preoperative pneumoperitoneum in the surgical preparation of complex eventrations

José Luis Díez Vigil ${ }^{1}$, Paula Barquero Dueñas², Mireia Domínguez Bastante ${ }^{1}$, Mohamed Hassin Mohamed Chiari ${ }^{1}$, Arturo Gómez Arroyo ${ }^{1}$, Alfonso Mansilla Roselló ${ }^{1}$

${ }^{1}$ Hospital Universitario Virgen de las Nieves. Granada (España). ${ }^{2}$ Facultad de Medicina. Universidad de Granada (España)

Recibido: 12-11-2020

Aceptado: 01-12-2020

Autor para correspondencia: José Luis Díez Vigil. Hospital Universitario Virgen de las Nieves. Av. de las Fuerzas Armadas, 2. 18014 Granada (España)

Correo electrónico: josediez81@gmail.com

DOI: $10.20960 /$ rhh.00389

Conflictos de interés: los autores declaran no tener conflictos de interés.

\section{RESUMEN}

Introducción: Las eventraciones complejas constituyen grandes defectos de la pared abdominal cuya reparación correcta supone un reto para el cirujano. El manejo de estos pacientes requiere una adecuada planificación para abordar el defecto con éxito. El neumoperitoneo preoperatorio progresivo (NPP) como herramienta 
terapéutica para preparar a estos pacientes ha permitido mejorar los resultados de esta cirugía y disminuir las complicaciones.

Objetivo: Demostrar la eficacia de la preparación preoperatoria con neumoperitoneo como complemento a la reparación quirúrgica de las eventraciones complejas.

Material y métodos: Estudio retrospectivo de 24 pacientes con eventración compleja entre abril de 2018 y enero de 2020, seleccionados previamente para la preparación preoperatoria del neumoperitoneo progresivo y a los que posteriormente se les realizó la técnica de reparación quirúrgica.

Resultados: Las complicaciones derivadas del uso del NPP fueron prácticamente nulas y fue bien tolerado por los pacientes. La preparación con esta técnica se llevó a cabo durante una media de 12 días antes de la cirugía y el volumen medio de aire administrado fue de $7979 \mathrm{~cm}^{3}$. Con este procedimiento se consiguió que no aumentase la presión intrabdominal tras la intervención quirúrgica y mantenerla en unos niveles óptimos, así como disminuir las complicaciones derivadas de su aumento. La adecuada aplicación de esta herramienta, junto a las técnicas quirúrgicas empleadas (TAR o RivesStoppa) y el uso de material protésico, facilitaron el tratamiento y la adecuada resolución de la eventración en todos los pacientes.

Conclusiones: EI NPP resulta ser una herramienta terapéutica segura y útil para hacer frente a este tipo de defectos de la pared abdominal. Sus inconvenientes son casi inexistentes y supone una gran ventaja en la resolución de las eventraciones.

Palabras clave: Neumoperitoneo, preoperatorio, eventración.

\section{ABSTRACT}

Introduction: Complex eventration produce great defects on the abdominal wall whose repair means a great challenge. A correct planning with patients is needed in order to tackle this defect with success. Progressive preoperative pneumoperitoneum as a 
therapeutic tool to prepare these patients has improved the results of this surgery and reduced complications.

Objective: The aim of this study is to demonstrate the efficacy of the pre-surgery preparation with pneumoperitoneum (PPP) as a complement to surgical reparation of the complex eventrations.

Material and methods: Retrospective study of 24 patients with complex eventration, within April 2018 and January 2020, previously chosen to pre-surgery infiltration of pneumoperitoneum and that afterwards had the corresponding technique of surgical reparation applied.

Results: Of all the studied cases, derived complications of using the PPP were almost inexistent and it was well tolerated by patients. The preparation with this technique took place throughout approximately 12 days before surgery and the amount of air provided was about $7,979 \mathrm{~cm}^{3}$. With that, the rise of the intraabdominal pressure after surgery is minimum, keeping it in optimal levels and decreasing possible complications. The correct application of this tool, the employed surgical techniques (TAR or Rives-Stoppa) and the use of prosthetic material have facilitated the success of the eventration's repair.

Conclusions: The PPP has turned out to be a safe and useful therapeutic tool to deal these kind of abdominal wall defects. Its issues are almost non-existent and it means a great advantage in the eventration's surgery.

Keywords: Pneumoperitoneum, preoperative, eventration.

\section{INTRODUCCIÓN}

La eventración es la protrusión o prolapso de un saco peritoneal, normalmente acompañado de estructuras anatómicas de la cavidad abdominal, como el epiplón o vísceras, a través de una apertura musculoaponeurótica patológica producida después de una cirugía ${ }^{1}$. La Sociedad Europea de la Hernia (EHS) las clasifica según su 
localización anatómica en eventraciones de la línea media (M) o laterales (L), y estas en subtipos: subxifoidea (M1), epigástrica (M2), umbilical (M3), infraumbilical (M4) y suprapúbica (M5); y las laterales en cuatro: subcostal (L1), flanco (L2), ilíaca (L3) y lumbar (L4). A su vez, según el diámetro transverso considera W1 $(<4 \mathrm{~cm})$, W2 $(4-10$ $\mathrm{cm}$ ) y W3 (> $10 \mathrm{~cm}$ ). Por último, si la eventración es recidivada se denomina R1 y en caso contrario, $\mathrm{RO}^{2,3}$.

Las eventraciones son grandes defectos parietales que conllevan la retracción de los músculos laterales del abdomen y su engrosamiento, lo que disminuye su longitud y la capacidad de la pared abdominal de contener las vísceras y provoca la herniación a través de dicho defecto del peritoneo recubierto por la piel y cuyo contenido puede estar formado por distintas vísceras abdominales.

Las «hernias con pérdida de derecho a domicilio» se definen como aquellas en las que más del $50 \%$ del contenido de la cavidad abdominal se encuentra fuera de esta, forma parte del saco herniario ${ }^{4}$ y hay una presencia continua en el saco herniario de una parte significativa del paquete intestinal, con el consiguiente riesgo de estrangulación ${ }^{5}$. Por tanto, la integridad de la pared permite mantener una presión intraabdominal positiva y, con ello, una adecuada dinámica de los distintos órganos y aparatos. Si esta integridad de la pared se pierde, lo que da lugar a eventraciones complejas, se producen una serie de alteraciones fisiológicas (posturales, deambulatorias, respiratorias, circulatorias, defecatorias, miccionales, etc.), como en la calidad de vida de los pacientes (repercusiones económicas, psicológicas, cuadros suboclusivos de repetición, etc. $)^{5}$.

La incidencia de eventraciones es de un 13-20\% en pacientes con laparotomía previa ${ }^{6,7}$, sobre todo en los 3 años siguientes a la cirugía. Constituyen un reto en cuanto a su reparación, pues el $30 \%$ de las eventraciones reparadas sin material protésico recidivan debido, sobre todo, a la elevada tensión intraabdominal que se genera ${ }^{6}$. En su tratamiento se persigue la aproximación de las estructuras musculares sin tensión. En el caso de las «eventraciones con pérdida 
de derecho a domicilio», la reducción del contenido herniado tras la reparación quirúrgica puede provocar una alteración de la ventilación pulmonar y hemodinámica del paciente por el aumento de la presión intraabdominal, lo que puede desencadenar el síndrome compartimental abdominal ${ }^{8}$. Habitualmente son pacientes considerados inoperables, con una mortalidad del $1 \%$ y una morbilidad del $10 \%$.

El objetivo del tratamiento quirúrgico es el cierre del defecto aponeurótico mediante la aproximación fascial primaria y, si esto no fuera posible, mediante la colocación de un material protésico. En ocasiones no es posible la aproximación adecuada del defecto aponeurótico, por lo que se recomienda conseguirlo mediante técnicas de separación de componentes (TSC). La TSC tradicionalmente más usada ha sido la descrita por Ramírez ${ }^{9}$, pero provoca un gran número de complicaciones de la herida, desde un $26 \%$ hasta un $63 \%{ }^{10}$. Existen otras técnicas que permiten la disección del espacio preperitoneal y que reducen las complicaciones asociadas al TSC. La técnica de Rives-Stoppa consigue la creación de un espacio preperitoneal en el que se coloca una malla de polipropileno (PPL) entre los vientres musculares del músculo recto y la fascia posterior de dicho músculo ${ }^{9,10}$. Sin embargo, esta técnica no permite una disección más allá del límite lateral de la vaina posterior del músculo recto. La separación posterior de componentes (SPC) lleva a cabo una separación de los músculos oblicuo menor o interno y transverso, lo que crea un espacio lateral por el que poder extender la malla de PPL ${ }^{11}$. En 2012, Novitsky describió una técnica alternativa denominada TAR (transversus abdominis release), de elección en eventraciones de más de $10 \mathrm{~cm}$ de tamaño transversal ${ }^{12}$ para acceder a un espacio preperitoneal más amplio9-13. Existe una modificación de esta técnica que aporta como ventaja una mayor extensión del espacio preperitoneal, lo que podría suponer una menor tasa de recidivas y de complicaciones ${ }^{12}$. 
El uso del neumoperitoneo preoperatorio progresivo (NPP) ha demostrado ser una técnica útil y eficaz para el tratamiento de grandes defectos de la pared abdominal ${ }^{14}$. Fue descrito por primera vez por Iván Goñi Moreno en 194015,16. Desde 1940 hasta la actualidad se han producido numerosas modificaciones de esta técnica, con variaciones en la forma de la punción, el tipo de catéter o el gas utilizados $^{15}$. En su técnica original, se coloca un catéter intraperitoneal y se pasa aire ambiente «lavado» con yodopovidona para reducir el riesgo de infecciones, con un sistema de sifón de dos frascos. En 1954 Koontz and Graves introdujeron el procedimiento en Estados Unidos. En 1990 Caldironi realizó punciones diarias con aguja de Veress utilizando $\mathrm{CO}_{2}$. En 1996 Naslound usó un Port-a-Cath ${ }^{\circledR}$. En 1997 Bevawi empleó un catéter de Tenckhoff. Por último, en 2001 Martínez Munive utilizó un catéter subclavio de doble luz y solo aire ambiente, sin filtros, pues el $\mathrm{CO}_{2}$ y el oxígeno que se utilizaban inicialmente en esta técnica presentaban una absorción 4 veces más rápida en el espacio intraperitoneal que el aire ambiente ${ }^{15}$.

Los objetivos que persigue el NPP son: disección neumática de bridas, que facilita la reintroducción de las asas intestinales en la cavidad abdominal ${ }^{5}$; distensión progresiva de la pared abdominal para poder cerrar el defecto herniario con menor tensión ${ }^{5}$; aumento volumétrico de la cavidad abdominal para facilitar la reintroducción visceral ${ }^{5}$; debido a la irritación peritoneal que crea, conlleva una vasodilatación con aumento de los macrófagos y una mejora de la capacidad cicatricial $^{5}$; regularización de la función respiratoria al prestar apoyo al diafragma en sus movimientos ${ }^{5}$, y mejora de la circulación portal, mesentérica e intestinal una vez que regresan paulatinamente las vísceras a la cavidad abdominal ${ }^{15}$ y se estabilizan la forma y la función diafragmáticas, lo que mejora la función ventilatoria ${ }^{17}$.

Nuestro objetivo primario con este trabajo es evaluar la efectividad de la aplicación prequirúrgica del neumoperitoneo en pacientes con eventraciones complejas con pérdida de derecho a domicilio. Como objetivos secundarios intentamos comprobar los beneficios del 
neumoperitoneo preoperatorio progresivo en la reparación quirúrgica de la eventración y estudiar los efectos adversos y las complicaciones derivadas de su administración.

\section{PACIENTES Y MÉTODOS}

Estudio retrospectivo de 24 pacientes consecutivos (23 casos de eventración compleja y un caso de hernia inguinoescrotal gigante con pérdida de derecho a domicilio), diagnosticados y operados entre abril de 2018 y enero de 2020. Los pacientes fueron seleccionados previamente para realización de NPP y, posteriormente, se realizó la reparación del defecto de la pared abdominal mediante técnicas de eventroplastia, ingresados en el Servicio de Cirugía General y del Aparato Digestivo del Hospital Universitario Virgen de las Nieves (Granada, España). En el estudio se incluyeron los pacientes con eventración de la pared abdominal de tamaño igual o superior a 10 cm (W3) o índice de Tanaka superior al $20 \%$. Se excluyeron del estudio los menores de 18 años, embarazadas, personas con enfermedad terminal médica o quirúrgica, con insuficiencia hepática o renal crónica, con obesidad mórbida, fumadores activos en el momento de la intervención o que rehusaron su participación en el estudio.

A los pacientes se les explicó ampliamente el procedimiento y los días que se consideraban convenientes de insuflación en función del tamaño y del volumen del saco herniario. En la historia clínica se puso especial énfasis, entre los antecedentes personales, las patologías asociadas (hipertensión arterial, diabetes mellitus, obesidad, hábitos tóxicos, etc.), así como entre los antecedentes quirúrgicos la existencia de reparaciones previas de la eventración. Todos los pacientes fueron valorados por el Servicio de Anestesia y por aquellos otros que se consideraron necesarios en función de las comorbilidades. Para el cálculo de la posibilidad de complicaciones derivadas de los problemas relacionados con la herida quirúrgica se 
utilizó el índice CEDAR (Carolinas Equation for Determining Associated Risks).

A todos los pacientes se les realizó un TAC para valorar las características de la eventración con el objetivo de estudiar las características de la musculatura lateral y de los rectos abdominales ${ }^{17}$, además de valorar con exactitud el contenido del saco herniario y de la cavidad abdominal, lo que proporciona una idea clara del tamaño del defecto ${ }^{5}$, ya que permite calcular la proporción de contenido abdominal herniado utilizando el índice de Tanaka ${ }^{17}$, el cual, al considerar el defecto una elipsoide, a partir de los tres diámetros espaciales obtiene cálculos volumétricos aproximados tanto del saco herniario como de la cavidad abdominal. Cada paciente firma dos consentimientos informados: uno para la administración del NPP y otro para la intervención quirúrgica. Antes de ser incluidos en el registro de demanda quirúrgica, se les informa acerca del procedimiento y de los tiempos a seguir, del tratamiento a administrar, de los efectos terapéuticos y de la posibilidad de efectos secundarios y de complicaciones. Tras ofrecer la formación facilitada, se constata que el paciente ha entendido bien lo expuesto y se han solventado las dudas que haya podido tener.

\section{PROCEDIMIENTO DE NEUMOPERITONEO}

En nuestro estudio, la inserción del catéter para introducir el aire ambiente en la cavidad abdominal se realiza según la técnica de Seldinger: mediante punción directa en el abdomen bajo anestesia local y guiada con ecografía, dejando el catéter en posición intraabdominal $^{5}$. Se señala el punto para realizar la punción, preferentemente en la línea media clavicular izquierda a nivel del hipocondrio izquierdo, por debajo del reborde costal. Con el paciente en decúbito dorsal bajo protocolo de asepsia, antisepsia y la aplicación de anestesia local ${ }^{18,19}$, se realiza la colocación del catéter. Se continúa colocando una llave de tres vías en la luz distal del catéter y se inicia la insuflación de la cavidad con aire ambiente con 
una cantidad de entre 500 y $1000 \mathrm{~cm}^{3}$ en función de la tolerancia del paciente. Antes de proseguir, para comprobar la presencia del adecuado neumoperitoneo, se realiza una radiografía de tórax en bipedestación. Si no hay complicaciones, el paciente es dado de alta al día siguiente tras insuflarle unos $1200 \mathrm{~cm}^{3}$ de aire ambiente. Va introduciéndose un volumen de $1200 \mathrm{~cm}^{3}$ al día de aire ambiente de forma ambulatoria, según la tolerancia del paciente. Se consigue así la distensión de la musculatura lateral del abdomen para después realizar la reparación quirúrgica y evitar la hipertensión intraabdominal ${ }^{20}$. El paciente sigue una estrecha revisión: en una hoja específica de evolución se anotan el volumen de aire diario insuflado y las incidencias que puedan ocurrir (molestias que sugieran alguna complicación, como infección o lesión visceral).

\section{TÉCNICA QUIRÚRGICA}

El procedimiento quirúrgico de elección es la reparación del defecto aponeurótico y la reconstrucción de la pared abdominal con material protésico. La técnica elegida se adapta a las características del paciente. Las mallas utilizadas fueron de polipropileno (PPL) o de fluoruro de polivinilideno (PDVF), en algunos casos asociadas con una biosintética reabsorbible (BIO-A $\left.{ }^{\circ}\right)$ y normalmente colocadas en posición preperitoneal o retromuscular. En los casos con defectos inferiores a $10 \mathrm{~cm}$ (W2) en los que era previsible el cierre del defecto mediante aproximación fascial primaria sin tensión, se llevó a cabo la técnica de Rives-Stoppa, mientras que en los que no era posible al ser superior a $10 \mathrm{~cm}$ (W3) se optó por la técnica TAR.

En la técnica de Rives-Stoppa se realiza una disección de la aponeurosis posterior del recto abdominal hasta la línea semilunar lateralmente; del triángulo graso de Conze cranealmente; del espacio de Retzius caudalmente y del espacio de Bogros laterocaudalmente, sobrepasando el pubis y los ligamentos de Cooper. Con ello se consigue la creación de un espacio preperitoneal en el que se coloca la malla entre los vientres musculares del músculo recto, la fascia 
posterior de dichos músculos y el espacio preperitoneal ${ }^{11,12}$. Con la utilización de esta técnica se disminuye la disección del tejido subcutáneo, pero no permite una disección más allá del límite lateral de la vaina posterior del músculo recto y no da solución a las eventraciones laterales ni a grandes defectos que sobrepasen estos límites ${ }^{13}$.

La técnica TAR es adecuada en eventraciones de más de $10 \mathrm{~cm}$ de tamaño transversal ${ }^{13}$. Se abre la fascia de los músculos rectos y se deja un colgajo anterior y otro posterior. Se diseca el espacio retromuscular de los rectos, el espacio de Retzius y los dos espacios laterales de Bogros hasta identificar los ligamentos de Cooper. Se identifica el ligamento arqueado por donde se inicia la sección del músculo transverso en ambos lados hasta el plano posterior de las últimas costillas y el espacio retroxifoideo/prediafragmático. Seguidamente, se suturan los defectos que existan del peritoneo y se hace una disección roma del espacio preperitoneal lateral. Se revisa la hemostasia y se cierra la fascia posterior del recto abdominal usando una parte del colgajo posterior que se dejó al principio. En este espacio, se coloca una primera malla de BIO-A ${ }^{\circledR}$ de la medida que mejor se adapte para cada paciente. Sobre ella, se coloca una segunda malla de PPL o PDVF que se fija a los ligamentos de Cooper caudalmente y a la línea alba o tendón central del diafragma a nivel craneal. Se deja un drenaje de tipo Blake en el espacio preperitoneal. Después de la colocación del material protésico, se reinserta el músculo transverso a la malla de PPL o PDVF, se revisa la hemostasia y se cierra la fascia anterior de los músculos rectos. Es posible que no pueda llegarse a una aproximación total de la fascia anterior de ambos rectos, por lo que el defecto fascial se recubrirá con parte del colgajo anterior creado previamente con el saco herniario, dejando siempre la malla cubierta o con tejido fascial o peritoneal. Para finalizar, se coloca un drenaje de tipo Redon en el espacio subcutáneo y se continúa con el cierre del tejido celular subcutáneo y de la piel. 


\section{VARIABLES DEL ESTUDIO}

Para la realización del estudio se construye una tabla con los 24 pacientes candidatos a cirugía de eventración del Hospital Universitario Virgen de las Nieves (fig. 3). En ella se recogen las variables definidas para cada paciente, que son de cuatro tipos:

1. Dependientes de las características del paciente: edad, género, IMC, HTA, DM, tamaño de la eventración, tipo de eventración, índice de Tanaka, índice ASA, índice CEDAR, tabaquismo y consumo de alcohol.

2. Dependientes del procedimiento del neumoperitoneo: dolor, irritación frénica, enfisema subcutáneo, sensación de plenitud gástrica, saciedad temprana, derrame pleural, atelectasia, infección, imposibilidad de encontrar un acceso seguro para realizar la punción o lesión visceral ${ }^{4}$.

3. Dependientes de la intervención quirúrgica: técnica quirúrgica empleada, tipo de malla, presión intrabdominal preoperatoria ( $\mathrm{cm} \mathrm{H}_{2} \mathrm{O}$ ), presión intrabdominal posoperatoria $\left(\mathrm{cm} \mathrm{H}_{2} \mathrm{O}\right)$, duración del procedimiento, puente aponeurótico $(\mathrm{cm})$ y complicaciones quirúrgicas.

4. Dependientes del resultado del tratamiento: estancia hospitalaria, infección de la herida quirúrgica y reintervención quirúrgica.

\section{SEGUIMIENTO}

Tras el alta, todos los pacientes son citados en consulta un mes después de la intervención y posteriormente al sexto mes, al año y a los años. Se constata tanto la evolución de los pacientes como las posibles complicaciones surgidas durante todo el procedimiento.

\section{RESULTADOS}

Del total de los 24 pacientes del estudio, 16 fueron mujeres (66.66 \%) y 8 , hombres (33.33\%). La edad media fue de 61 años, con un intervalo de edades de entre 24 y 78 . El IMC medio, de 34 (mínimo de 24 y máximo de 48). En la clasificación anestésica, 2 pacientes fueron 
ASA I (8.3 \%); 10, ASA II (41.6\%) y 12 pacientes, ASA III (50\%). En cuanto a las comorbilidades, 12 fueron hipertensos (50\%); 8, diabéticos (33\%); 6, fumadores (25\%) y 2 consumían alcohol (8.4\%). En cuanto a la recidiva previa, 12 de las eventraciones eran recidivadas ( $50 \%$ ). Un $95.83 \%$ de los casos (23 pacientes) habían sido intervenidos previamente y en 9 de ellos (39.13\%) la cirugía previa se había debido a defectos herniarios y eventraciones. Tan solo un paciente no presentaba ningún tipo de intervención previa $(4.16 \%)$, el mismo paciente que presentaba la hernia inguinoescrotal derecha gigante.

Considerando el saco herniario y el abdomen como estructuras elipsoides, a partir de los tres diámetros espaciales y aplicando la fórmula de volume ratio $^{17}$, se obtiene un volumen aproximado del defecto. A cada paciente se le hizo un TAC (figs. 1-3) para calcular el índice de Tanaka. El valor medio del índice de Tanaka fue de $27.5 \%$ (rango 4-85\%).

El índice CEDAR tuvo un valor medio de $32.9 \%$ (mínimo de $16 \%$ y máximo de $71 \%$ ).

Las características de las eventraciones fueron las siguientes: el tamaño de la eventración fue de un diámetro longitudinal medio de $15.3 \mathrm{~cm}$ (mínimo de $8 \mathrm{~cm}$ y máximo de $30 \mathrm{~cm}$ ); el diámetro transversal medio, de $11.94 \mathrm{~cm}$ (mínimo de $7.7 \mathrm{~cm}$ máximo de 20 $\mathrm{cm}$ ); el área prequirúrgica, de $147.78 \mathrm{~cm}^{2}$ (mínimo de $52 \mathrm{~cm}^{2}$ y máximo de $392.7 \mathrm{~cm}^{2}$ ) y el volumen del saco herniario, de $2253.2 \mathrm{~cm}^{3}$ (mínimo de $473 \mathrm{~cm}^{3}$ y máximo de $7176 \mathrm{~cm}^{3}$ ). Un paciente fue operado por hernia escrotal gigante $(4.16 \%)$ con pérdida de derecho a domicilio. De las eventraciones $(n=23), 20$ de ellas, un $83.33 \%$, se localizaban en la línea media (M1-M5), mientras que las restantes, 3 de ellas (12.5\%), eran laterales (L1-L4). En cuanto al tamaño, un $78.26 \%$ (18 casos) era W3 (> $10 \mathrm{~cm}$ ) y un $21.7 \%$ (5 casos) era W2 (> 4-10 cm) (tabla I).

En 13 de los participantes $(54.2 \%)$ se realizó tratamiento preoperatorio con toxina botulínica, además del empleo de 
neumoperitoneo, con un promedio de 12 días (mínimo de 6 y un máximo de 24 días). El volumen de aire administrado fue de $7979 \mathrm{~cm}^{3}$ (mínimo de $4100 \mathrm{~cm}^{3}$ y un máximo de $15000 \mathrm{~cm}^{3}$ ). Tan solo se produjo un efecto adverso significativo (enfisema subcutáneo) en un paciente $(4.2 \%)$, que se resolvió de manera conservadora. En un $95.8 \%$ de los pacientes del estudio no hubo complicaciones derivadas del procedimiento.

La duración de la operación fue de 180 minutos (mínimo de 70 y máximo de 450 minutos). En cuanto a las técnicas que se llevaron a cabo fueron: en 10 casos, TAR (41.66 \%), dos de las cuales fueron TAR derecha. En un paciente, además del TAR se realizó la reconstrucción del tránsito. En 12 pacientes se llevó a cabo la técnica de RivesStoppa (50\%), incluyendo en dos de ellos, además, abdominoplastia. En un paciente se realizó conjuntamente el tratamiento de fístula vesicovaginal. En el paciente con hernia inguinal se realizó una hernioplastia por vía anterior según la técnica de Lichstenstein (4.16\%). Los tipos empleados de malla fueron: BIO-A®, PPL, PDVF y Progrip ${ }^{\circledR}$ (tabla II). En un caso se empleó malla de PPL autoadhesiva (Progrip $®)$ ( $4.16 \%)$; en cinco, PPL (20.83\%); en nueve casos, BIO$A \circledR+P P L \quad(37.5 \%)$; en otros nueve, BIO-A®+PDVF (37.5\%). Su tamaño se individualizó en cada caso según el tamaño de la eventración y el tamaño puente. La PIA preoperatoria fue de 12.1 $\mathrm{cmH}_{2} \mathrm{O}$ (8 $\mathrm{cmH}_{2} \mathrm{O}$ mínimo y $19 \mathrm{cmH}_{2} \mathrm{O}$ máximo), mientras que la PIA tras la intervención quirúrgica fue de $12.5 \mathrm{cmH}_{2} \mathrm{O}\left(9 \mathrm{cmH}_{2} \mathrm{O}\right.$ mínimo y $18 \mathrm{cmH}_{2} \mathrm{O}$ máximo). El área del defecto aponeurótico tras la intervención quirúrgica fue de $8.5 \mathrm{~cm}^{2}$ (mínimo de $0 \mathrm{~cm}^{2}$ y máximo de $93.5 \mathrm{~cm}^{2}$ ). En 15 pacientes $(62.5 \%)$ se consiguió aproximar por completo la vaina de los rectos, con un cierre completo del defecto (tabla III).

Con respecto a las complicaciones posquirúrgicas, se presentaron en 6 pacientes (25\%): 3 infecciones de la herida quirúrgica (12.5\%) del total de pacientes, un caso de sepsis por $E$. coli (4.16\%), un hematoma intraabdominal $(4.16 \%)$ y un caso que se reintervino por 
perforación intestinal (4.16\%). La estancia hospitalaria media fue de 10 días, desde un mínimo de 2 a un máximo de 45 días. Hubo 4 complicaciones tardías ( $16.66 \%$ ) tras la cirugía que precisaron reingreso hospitalario: dos suboclusiones intestinales $(8.33 \%)$ que recibieron tratamiento médico, un caso de obstrucción intestinal por bridas que requirió reintervención quirúrgica (4.16\%) y un absceso de pared abdominal (4.16\%). Tras el alta, los pacientes acudieron a revisión al mes, tras el que no hubo ninguna incidencia: el $100 \%$ de los casos evolucionó sin complicaciones ni recidivas. Al sexto mes acudieron a revisión 15 pacientes $(62.5 \%$ ), de los que el $100 \%$ continuó evolucionando favorablemente. Al año se revisaron 5 pacientes (20.8\%), sin evidencia de recidiva de la eventración.

\section{DISCUSIÓN}

Se define como hernia o eventración abdominal con pérdida de domicilio a la entidad en la que más del $50 \%$ del contenido de la cavidad abdominal se encuentra fuera de esta ${ }^{4}$. El NPP está indicado como paso previo a la reparación quirúrgica para casos seleccionados de patología herniaria en los que existe una pérdida de derecho a domicilio ${ }^{5}$. Esta técnica fue descrita por primera vez por Goñi Moreno en $1940^{15,16}$. Su forma de administración ha ido progresando durante más de 60 años, perfeccionándose la técnica y extendiéndose su empleo en cirugías de pared abdominal gracias a los beneficios que proporciona ${ }^{5,21}$. Se ha convertido en una herramienta terapéutica muy útil y que continúa dándose a conocer hoy día tras los buenos resultados obtenidos en pacientes intervenidos de eventraciones complejas.

Las eventraciones resultan ser un defecto en la pared abdominal que va creciendo y alterando progresivamente su fisiología normal, hasta la incapacidad para devolver el contenido del saco herniario al interior de la cavidad abdominal, ya que existe una gran desproporción entre el continente y el contenido ${ }^{20}$. Esto afecta también al equilibrio respiratorio, lo que provoca restricción tanto inspiratoria como 
espiratoria y la disminución del retorno venoso en cava y porta, así como del retorno linfático ${ }^{21}$. El intestino y el mesenterio se edematiza por la compresión que ejerce el anillo herniario fibrótico. En casos avanzados, la piel distendida en exceso se atrofia por hipovascularización y pueden aparecer ulceraciones y sobreinfecciones de los pliegues ${ }^{21}$. Por tanto, además de las repercusiones fisiológicas (posturales, deambulatorias, respiratorias, circulatorias, defecatorias, miccionales, etc.), también tienen importantes repercusiones en la calidad de vida (económicas, psicológicas, cuadros suboclusivos de repetición, etc. $)^{5}$.

Nuestra serie incluye defectos de tamaño igual o superior a $10 \mathrm{~cm}$ (W3) o índice de Tanaka mayor de $20 \%$. Para abordar estos defectos complejos son necesarias tanto medidas higiénicas y dietéticas por parte del paciente como una preparación previa específica que permita mejorar la capacidad abdominal gracias al NPP.

En cuanto a los factores modificables por parte del paciente, se encuentra la adopción de estilos de vida saludables, ya que algunos de ellos se han relacionado con una mayor incidencia de eventración, como el IMC elevado y el tabaquismo ${ }^{5}$. La abstinencia del tabaco es fundamental. En nuestro estudio, un $25 \%$ lo eran. Además, existen otros factores potenciales de riesgo, como la infección de la herida, que es el principal factor que impide la correcta cicatrización, es causa de evisceración y tiene un papel importante en la aparición de eventraciones (está implicada en el $17-50 \%$ de los fallos de pared abdominal y se considera que incrementa la tasa de eventración hasta el $40 \%)^{5}$.

Respecto a la edad, aunque algún estudio concluye que una edad superior a los 45 años es un factor de riesgo en el desarrollo de eventración, los resultados son contradictorios. En cuanto al sexo, aunque hay más dehiscencias entre los varones, parece que la tasa de eventración es la misma. Las enfermedades sistémicas severas (diabetes, insuficiencia renal, enfermedad cardíaca, EPOC, etc.) son factores de riesgo en la aparición de complicaciones porque 
aumentan la probabilidad de que el material de sutura rasgue los tejidos debido a su debilitamiento.

Las intervenciones quirúrgicas sobre el tracto gastrointestinal de larga duración, las urgentes o las reintervenciones aumentan la tasa de dehiscencia de la pared abdominal al estar asociadas a una elevada frecuencia de infección de la herida quirúrgica ${ }^{5}$, de ahí la importancia de la preparación preoperatoria con una historia clínica completa, con especial énfasis en la descripción de las intervenciones quirúrgicas anteriores a la eventración, de la valoración de las patologías asociadas que precisarán control exhaustivo y de intentar modificar los factores de riesgo antes de la intervención a través de la pérdida de peso, del abandono del tabaquismo, de la fisioterapia respiratoria o del control adecuado de la glucemia en pacientes diabéticos.

El NPP es uno de los métodos previos que permite una expansión progresiva de la musculatura abdominal para posteriormente poder reparar el defecto herniario con la menor tensión muscular posible y un adecuado reintegro de las vísceras al abdomen, con lo que se reduce la posibilidad de complicaciones derivadas de una hiperpresión abdominal ${ }^{21}$, aumenta progresivamente la capacidad abdominal, evita la elevación brusca del diafragma y facilita la circulación venosa de retorno. Además, a partir de la segunda semana estimula el sistema inmunitario y mejora la respuesta celular de los macrófagos, lo que favorece la posterior cicatrización de las heridas ${ }^{20}$. En nuestro estudio, la cantidad media de neumoperitoneo que se ha administrado ha sido de unos $7979 \mathrm{~cm}^{3}$, repartidos en unos 11 días. No obstante, la cantidad de neumoperitoneo se ha empleado de una forma individualizada en función del tamaño de la eventración y de la tolerancia. La mayoría de los estudios describe el uso del NPP para la reparación de las eventraciones gigantes; sin embargo, también puede utilizarse en hernias inguinales y umbilicales gigantes con buenos resultados ${ }^{19,20,22,23}$, como hicimos en un caso con una hernia inguinoescrotal gigante. 
Las complicaciones descritas para esta técnica son principalmente locales, como el enfisema subcutáneo y las infecciones de la pared abdominal. Las complicaciones graves son poco frecuentes ${ }^{20}$. En nuestra experiencia, los efectos secundarios derivados de la técnica han sido prácticamente nulos. Tan solo un paciente presentó un enfisema subcutáneo que se resolvió de manera conservadora. Cabe destacar que en 13 casos se aplicó también, junto al neumoperitoneo, toxina botulínica para relajar la musculatura abdominal. Puede deducirse que ambas técnicas se complementan y proporcionan beneficios en ambos casos, pues también se ha defendido el uso de TBA y ya se ha empleado debido a sus buenos resultados en grandes defectos herniarios ${ }^{6,15}$.

La presión intraabdominal preoperatoria y la posoperatoria son los aspectos más importantes de la cirugía, ya que debe existir la menor diferencia posible entre ellas para evitar así un aumento excesivo de la presión que conlleve complicaciones. La PIA es el estado de presión que hay en la cavidad abdominal determinada por el índice de masa corporal, la posición, la actividad muscular de la pared y la respiración ${ }^{22}$. Depende del volumen de los órganos macizos y huecos, de cuánto y cómo se ocupe la cavidad abdominal o el retroperitoneo (aire, sangre, edema, packing, etc.) y de la distensibilidad de la pared $^{22}$. Al tratarse de una cirugía con cierre de pared a tensión, es obligatorio su control estricto, pues valores elevados mantenidos pueden dar lugar a un SCA con repercusiones pulmonares, cardiovasculares, renales y en la circulación esplácnica. La variabilidad de estas presiones ha sido mínima en el estudio y siempre se ha mantenido por debajo del valor límite que considera hiperpresión abdominal: $15 \mathrm{cmH}_{2} \mathrm{O}^{22}$. Se confirma, por tanto, uno de los beneficios del uso del neumoperitoneo: permite un cierre de pared con la mínima tensión posible, con lo que así se reduce así la probabilidad de un aumento de PIA tras la reparación quirúrgica.

Los resultados han sido muy buenos gracias tanto a la adecuada preparación previa a la cirugía como a las técnicas quirúrgicas 
empleadas, que también han demostrado su gran utilidad en cirugías de pared abdominal (Rives-Stoppa, TAR, etc.) ${ }^{11,12}$. El procedimiento quirúrgico se adaptó a las características de cada paciente. En los casos que era posible una aproximación del defecto aponeurótico sin o con muy poca tensión, se llevó a cabo la técnica de Rives-Stoppa. Por el contrario, en los casos que presentaban una separación más amplia del defecto o una tensión más elevada al cierre, se optó por la técnica TAR. Aunque no pudo cerrarse en todos los casos, el área de la eventración sí se redujo de forma significativa un $94 \%$.

Tal y como se ha descrito anteriormente, solo se produjeron complicaciones inmediatas a la cirugía en un $25 \%$, de las que la mitad fueron infecciones de la herida (posible complicación frecuente en cualquier tipo de cirugía).

A la vista de los resultados del estudio se demuestra y se corrobora con los estudios ya existentes que los beneficios del NPP superan con creces los inconvenientes de su empleo y que estos últimos son prácticamente nulos. Recordemos que los objetivos que perseguíamos con esta técnica ${ }^{5,17,21}$ eran: disección neumática de bridas y adherencias, distensión progresiva de la pared abdominal para reintroducir más fácilmente las asas intestinales y conseguir un cierre herniario con la menor tensión posible, reducir el riesgo de aumento excesivo de la PIA y sus complicaciones derivadas y asegurar una cirugía con mejores resultados y menos complicaciones posoperatorias, con lo que se consigue un mayor bienestar del paciente y una menor estancia hospitalaria. Todo esto se consigue gracias, en parte, a una adecuada preparación prequirúrgica, lo que delata la importancia y la utilidad del NPP como herramienta terapéutica en este tipo de defectos herniarios ${ }^{19,23,24}$.

Por tanto, la preparación previa de los pacientes con NPP, combinada en ocasiones con otras técnicas como la TBA, junto con los métodos quirúrgicos que se llevan a cabo, proporcionan las condiciones óptimas para obtener una adecuada restitución de la pared y una menor tasa de recidivas ${ }^{14,25}$. En definitiva, las ventajas de esta técnica 
superan, sin ninguna duda, a los inconvenientes. El uso del neumoperitoneo permitió la reparación de los defectos herniarios sin producir en ningún caso síndrome compartimental y sin manifestar, tras la revisión, ninguna recidiva.

\section{CONCLUSIONES}

Podemos considerar el NPP un complemento seguro y eficaz para abordar los grandes defectos de la pared abdominal, como las eventraciones complejas. Este método incrementa tanto el volumen de la cavidad abdominal como la longitud de los músculos abdominales, lo que permite una reparación con la mínima tensión posible. A su vez, reduce notablemente los riesgos preoperatorios y posoperatorios en las hernias gigantes, ya que, además del incremento de volumen abdominal, eleva la tolerancia del paciente a la cirugía, disminuye el riesgo de síndrome compartimental y de dehiscencia y reduce la tasa de recurrencias.

\section{BIBLIOGRAFÍA}

1. Tripoloni D. Eventraciones y evisceraciones abdominales. En: Giménez M, editor. Cirugía. Fundamentos para la práctica clínicoquirúrgica para la práctica clínico-quirúrgica. Primera edición. Buenos Aires: Editorial Médica Panamericana; 2014. p. 99-108.

2. Marenco B, Retamar M, Sánchez M, Guadalajara JF, Cano A, López JA, et al. Tipos de hernias. Clasificaciones actuales. Clasif actuales. Cir Andal. 2018;29(2):77-9.

3. Muysoms FE, Miserez M, Berrevoet F, Campanelli G, Champault GG, Chelala $E$, et al. Classification of primary and incisional abdominal wall hernias. Hernia. 2009;13(4):407-14. DOI: 10.1007/s10029-009-0518-x 4. Arias PM, Roque M, Pasarín MA, Albornoz PD, Cacciavillani G. Neumoperitoneo preoperatorio en eventración subcostal gigante. Rev Hispanoam Hernia. 2016;4(4):157-61. DOI: 10.1016/j.rehah.2016.07.001 
5. Morales S, Barreiro F, Hernández P, Feliu X. Cirugía de la Pared Abdominal. Guía Clínica de la AEC. 2.a ed. Madrid: Arán Ediciones; 2013 [citado 17 abril 2020]. p. 57-8; 80-1; 202-9.

6. Rodríguez G, Cruz A, Oña FM, García LR, Sánchez AA, Chávez UJ, et al. Separación de componentes química (toxina botulínica tipo A) en la reparación de hernia ventral planeada: un modelo murino. Rev Hispanoam Hernia. 2015;3(4):139-46. DOI: 10.1016/j.rehah.2015.04.002

7. De la Cuadra R. Hernia abdominal compleja. Rev Chil Cir. 2005;57(4):354-58.

8. Hernández López A. Tratamiento actual de grandes eventraciones con las técnicas de separación de componentes anteriores y posteriores. Rev Hispanoam Hernia. 2016;4(1):1-3. DOI: 10.1016/j.rehah.2015.11.003

9. Rivas J, Turiño J, Cabello A, Pérez M, Bayón A, Martínez A, et al. Separación posterior de componentes. Cir Andal. 2018;29(2):197-9.

10. Carbonell AM, Cobb WS, Chen SM. Posterior components separation during retromuscular hernia repair. Hernia. 2008;12(4):359-62. DOI: 10.1007/s10029-008-0356-2

11. Novitsky YW, Elliott HL, Orenstein SB, Rosen MJ. Transversus abdominis muscle release: a novel approach to posterior component separation during complex abdominal wall reconstruction. Am J Surg. 2012;4(5):709-16. DOI: 10.1016/j.amjsurg.2012.02.008

12. García MÁ, Miguel CS, Robin A, López J, Blázquez LA, Cruz D, Melero A. TAR (transversus abdominis release) y Madrid modification of TAR como técnicas alternativas en la reparación de la pared abdominal compleja. Transversus Abdominis Release in complex incisional hernia. Cir Andal. 2018;29(2):200-2.

13. Cuenca O, Ferreira R, Theys L, Martínez N, González E, Pérez R. Aplicación del neumoperitoneo progresivo preoperatorio en las afecciones parietales complejas. Rev Chil Cir. 2013;46(2):35-44. 
14. Mayagoitia JC, Carbonell F, Moreno-Egea A. Neumoperitoneo progresivo preoperatorio y uso de la toxina botulínica para el manejo de las hernias con pérdida de dominio. AEC. 2012;(27):365-75.

15. Moreno G. Chronic eventrations and large hernias. Preoperative treatment by progressive pneumoperitoneum. Original procedure. Surgery. 1947;22(6):945-53.

16. Bueno J, Torregrosa A, Jiménez R, Carbonell F, García P, Bonafé S, et al. Preparación preoperatoria de la hernia con pérdida de domicilio. Neumoperitoneo progresivo y toxina botulínica tipo A. Cir Esp. 2017;95(5):245-53. DOI: 10.1016/j.ciresp.2017.04.006

17. Tanaka EY, Yoo JH, Rodrigues AJ, Utiyama EM, Birolini D, Rasslan S. A computerized tomography scan method for calculating the hernia sac and abdominal cavity volume in complex large incisional hernia with loss of domain. Hernia. 2010;(14):63-9. DOI: 10.1007/s10029009-0560-8

18. Mayagoitia JC, Arenas JC, Suárez D, Díaz V, Álvarez R. Neumoperitoneo progresivo preoperatorio en hernias de la pared abdominal con pérdida de dominio. Cir Gen. 2005;27(4):280-5.

19. Flores JJ, de la Cruz LD, Reyes AC, Balderrama SC. Neumoperitoneo progresivo preoperatorio en hernias de pared abdominal. Cir Gen. 2019;41(2):92-7.

20. Sánchez M, Bazán $C$, Casado MD, Pérez $D$, Bengoechea $A$, Fernández JL. Hernias con pérdida de derecho a domicilio. Cir Andal. 2013;24(3-4):272-3.

21. Hernández A, Rubalcava JV. Infiltración de toxina botulínica en la preparación preoperatoria de las hernias con defectos de $10 \mathrm{~cm}$ (y hasta $15 \mathrm{~cm}$ ). Rev Hispanoam Hernia. 2016;4(2):43-9. DOI: 10.1016/j.rehah.2016.02.003

22. Dumont F, Fuks D, Verhaeghe P, Brehant O. Progressive pneumoperitoneum increases the lenght of abdominal muscles. Hernia. 2009;(13):183-7. DOI: 10.1007/s10029-008-0436-3 
23. Piskin T, Aydin C, Barut B. Preoperative progressive pneumoperitoneum for giant inguinal hernias. Ann Saudi Med. 2010(30)317-20. DOI: 10.4103/0256-4947.65268

24. Castellanos G, Piñero A, Fernández JA. La hipertensión intraabdominal y el síndrome compartimental abdominal: ¿qué debe saber y cómo debe tratarlos el cirujano? Cir Esp. 2007;81(1):4-11. DOI: 10.1016/S0009-739X(07)71249-6

25. Granel L, Gamón RL, Fortea C, Gómez F, Salvador JL. Neumoperitoneo preoperatorio para el tratamiento de la hernia inguinal gigante: revisión de nuestra experiencia. Rev Hispanoam Hernia. 2014;2(4):133-8.

26. Pamiés J, Aboud C, Navarro V. La valoración cualitativa y cuantitativa de la hernia abdominal con tomografía computarizada multidetector. Rev Hispanoam Hernia. 2013;1(4):149-58. DOI: 10.1016/j.rehah.2013.07.003

Tabla I. Clasificación de las eventraciones según la Sociedad Europea de Hernia (EHS)

\begin{tabular}{|l|l|l|l|}
\hline $\begin{array}{l}\text { N.o de } \\
\text { caso }\end{array}$ & Clasificación EHS & $\begin{array}{l}\text { Recidi } \\
\text { va }\end{array}$ & Intervenciones previas \\
\hline 1 & L1W3R1 & Sí & Colecistectomía \\
\hline 2 & M3M4M5W3 & No & Hernioplastia umbilical \\
\hline 3 & M3M4W2R1 & Sí & Hernioplastia umbilical \\
\hline 4 & M3M4M5W3R1 & Sí & Histerectomía \\
\hline 5 & M4M5W3 & No & Histerectomía \\
\hline 6 & M4M5W2 & No & Histerectomía \\
\hline 7 & M1-4W3R2 & Sí & Hernioplastia supraumbilical \\
\hline 8 & M3-4W3 & No & Colecistectomía \\
\hline
\end{tabular}




\begin{tabular}{|c|c|c|c|}
\hline & & & laparotomía por íleo biliar \\
\hline 9 & M2M3M4W3R1 & Sí & Hernia umbilical \\
\hline 10 & L3W3 & No & Trasplante renal \\
\hline 11 & M3M4W2 & No & Histerectomía, cesárea \\
\hline 12 & M3M4M5W3 & No & Histerectomía \\
\hline 13 & M3M4M5W3 & No & $\begin{array}{lll}\text { Laparotomía } & \text { por } & \text { hernia } \\
\text { diafragmática } & & \end{array}$ \\
\hline 14 & M3M4M5W3R1 & Sí & $\begin{array}{lll}\text { Colectomía } & \text { total } & \text { y } \\
\text { colecistectomía } & & \end{array}$ \\
\hline 15 & M1-5W3R1 & Sí & $\begin{array}{l}\text { Laparotomía por bridas y } \\
\text { resección de tumor de pared }\end{array}$ \\
\hline 16 & L4W2 & No & $\begin{array}{l}\text { Fractura del acetábulo, } \\
\text { injerto de pala iliaca }\end{array}$ \\
\hline 17 & M2M3W2R2 & Sí & $\begin{array}{lll}\text { Eventración trocar, } & \text { lap } \\
\text { eventroplastia } & & \\
\end{array}$ \\
\hline 18 & M2M3W3R1 & Sí & Hernioplastia umbilical \\
\hline 19 & M4-5W3 & No & Histerectomía \\
\hline 20 & $\begin{array}{l}\text { Hernia } \\
\text { inguinoescrotal } \\
\text { derecha gigante }\end{array}$ & No & \\
\hline 21 & M1M2M3W3R1 & Sí & 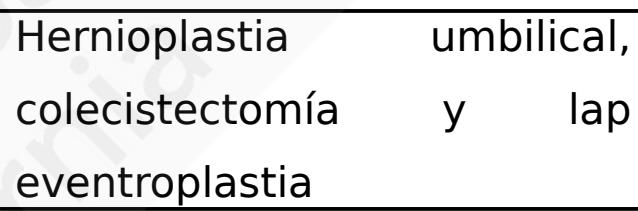 \\
\hline 22 & M2M3W3R1 & Sí & $\begin{array}{l}\text { Eventración trocar y lap } \\
\text { eventroplastia }\end{array}$ \\
\hline 23 & M3M4M5W3R2 & Sí & Histerectomía \\
\hline 24 & M4M5W3 & No & Colecistectomía, 3 cesáreas \\
\hline
\end{tabular}

Tabla II. Tipos de malla quirúrgica

\begin{tabular}{|c|c|c|c|c|}
\hline Tipo de malla & $\begin{array}{l}\mathrm{BIO}- \\
\mathrm{A} \otimes+\mathrm{PPL}\end{array}$ & $\begin{array}{l}\mathrm{BIO-} \\
\mathrm{A} \AA+P D V F\end{array}$ & PPL & $\begin{array}{l}\text { Progri } \\
p \AA\end{array}$ \\
\hline 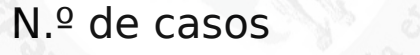 & 9 & 9 & 5 & 1 \\
\hline $\begin{array}{ll}\text { Porcentaje } & \text { de } \\
\text { casos }(\%) & \\
\end{array}$ & $37.5 \%$ & $37.5 \%$ & $\begin{array}{l}20.83 \\
\%\end{array}$ & $\begin{array}{l}4.16 \\
\%\end{array}$ \\
\hline
\end{tabular}

Tabla III. Variables pre- y poscirugía

\begin{tabular}{|l|ll|l|l|l|l|l|}
\hline Variab & $\begin{array}{l}\text { Tamaño } \\
\text { de }\end{array}$ & $\begin{array}{l}\text { Área } \\
\text { preciru }\end{array}$ & $\begin{array}{l}\text { Pre } \\
-\end{array}$ & $\begin{array}{l}\text { Tamaño } \\
\text { del }\end{array}$ & $\begin{array}{l}\text { Área de } \\
\text { la }\end{array}$ & $\begin{array}{l}\text { Pos } \\
-\end{array}$ \\
\hline
\end{tabular}




\begin{tabular}{|c|c|c|c|c|c|c|}
\hline \begin{tabular}{|l|} 
les \\
\end{tabular} & \begin{tabular}{|l} 
eventració \\
n (cm) \\
(diámetro \\
longitudin \\
al- \\
transversa \\
I)
\end{tabular} & $\begin{array}{l}\text { gía } \\
\left(\mathrm{cm}^{2}\right)\end{array}$ & $\begin{array}{l}\text { PIA } \\
\text { (cm } \\
\mathrm{H}_{2} \mathrm{O} \\
\text { ) }\end{array}$ & $\begin{array}{l}\text { puente } \\
\text { aponeurót } \\
\text { ico }(\mathrm{cm})\end{array}$ & $\begin{array}{l}\text { posciru } \\
\text { gía } \\
\left(\mathrm{cm}^{2}\right)\end{array}$ & $\begin{array}{l}\text { PIA } \\
\text { (cm } \\
\mathrm{H}_{2} \mathrm{O} \\
\text { ) }\end{array}$ \\
\hline \begin{tabular}{|l} 
Mínimo \\
\end{tabular} & $\begin{array}{l}\mathrm{L}=8 / \mathrm{T}= \\
7.7\end{array}$ & 52 & 8 & $\begin{array}{l}\mathrm{L}=0 / \mathrm{T}= \\
0\end{array}$ & 0 & 9 \\
\hline \begin{tabular}{|l} 
Máxim \\
o
\end{tabular} & $\begin{array}{l}\mathrm{L}=30 / \mathrm{T}= \\
20\end{array}$ & 392.7 & 19 & $\begin{array}{l}\mathrm{L}=17 / \mathrm{T} \\
=7\end{array}$ & 93.5 & 18 \\
\hline \begin{tabular}{|l} 
Valor \\
medio
\end{tabular} & $15.3 \times 11.9$ & 147.8 & $\begin{array}{l}12 . \\
1 \\
\end{array}$ & $2.13 \times 1.38$ & 8.5 & $\begin{array}{l}12 . \\
5 \\
\end{array}$ \\
\hline
\end{tabular}

